\title{
Thibauld Moulaert, Gouverner les fins de carrière à distance. Outplacement et vieillissement actif en emploi
}

Bruxelles, P.I.E-P. Lang, coll. « Action publique », n 9, 2012

Annie Jolivet

\section{OpenEdition}

Journals

Édition électronique

URL : http://journals.openedition.org/travailemploi/6784

DOI : 10.4000/travailemploi.6784

ISSN : $1775-416 \mathrm{X}$

Éditeur

DARES - Ministère du Travail

Édition imprimée

Date de publication : 1 octobre 2015

Pagination : 116-119

ISSN : 0224-4365

Référence électronique

Annie Jolivet, « Thibauld Moulaert, Gouverner les fins de carrière à distance. Outplacement et vieillissement actif en emploi », Travail et Emploi [En ligne], 144 | octobre-décembre 2015, mis en ligne le 01 octobre 2015, consulté le 22 septembre 2020. URL : http://journals.openedition.org/travailemploi/ 6784 ; DOI : https://doi.org/10.4000/travailemploi.6784 


\title{
Gouverner les fins de carrière à distance. Outplacement et vieillissement actif en emploi
}

\author{
Thibauld Moulaert
}

Bruxelles, P.I.E-P. Lang, coll. « Action publique », nº 9, 2012, 287 p.

\section{Lu par Annie Jolivet}

Thibauld Moulaert analyse dans cet ouvrage, tiré de sa thèse de sociologie soutenue en 2009, la transformation significative de l'action publique sur les fins de carrière à partir de l'exemple de l'outplacement' pour les 45 ans et plus mis en place en Belgique depuis 2005. Il le fait en combinant une réflexion critique sur les politiques dites de vieillissement actif et une analyse de l'outplacement « en train de se faire », largement fondée sur la sociologie pragmatique.

La première partie, relativement courte, est consacrée à l'émergence du concept de «vieillissement actif» en tant que nouveau référentiel de l'action publique. Le chapitre I rappelle la contribution des arguments démographiques à la construction, à partir du milieu des années 1990, d'une problématique du vieillissement dont l'un des enjeux est la redéfinition des parcours de fin de carrière, dans un contexte où les trajectoires tendent à se diversifier et à s'individualiser. Les relations entre politiques publiques et usagers sont dès lors réenvisagées : il s'agit de (re)donner aux individus les moyens d'agir (ce que reprend la notion d'empowerment) et de considérer les personnes comme des sujets (d'où la distinction entre subjectivation et individualisation $^{2}$ ). Le chapitre II resitue la notion de vieillissement actif/active ageing par rapport aux notions antérieures de successful ageing et de productive ageing ${ }^{3}$. Le concept de vieillissement actif, qui l'a emporté sur les autres à la fin des années 1990, combinerait le cœur de ces deux notions. La Commission européenne a toutefois plutôt insisté sur

\footnotetext{
* Centre d'études de l'emploi.

1. L'outplacement ou reclassement/replacement externe est un ensemble de services et de conseils fournis individuellement ou en groupe par un prestataire de services (public ou privé) afin de permettre à un travailleur soit de retrouver par lui-même et le plus rapidement possible un emploi, soit de développer une activité professionnelle en tant qu'indépendant (définition de l'Office national pour l'emploi de Belgique).

2. L'individualisation désigne la plus large place donnée à la décision de l'individu, qui en assume donc la responsabilité. La subjectivation introduit l'idée que l'individu se construit en tant que sujet à travers ses décisions, ses actions et ses relations avec d'autres.

3. La notion de successful ageing envisage le vieillissement réussi par opposition à l'idée que la vieillesse s'accompagne nécessairement d'un désengagement de la vie sociale. La notion de productive ageing, issue d'une évolution des réflexions dans le champ gérontologique, est utilisée « pour désigner toute activité d'un individu âgé qui produit des biens ou des services, payés ou non, ou développe la capacité à les produire ».
} 
la prolongation des carrières en considérant que le vieillissement actif désigne à la fois le fait de travailler plus longtemps, de prendre sa retraite plus tard et de rester actif après la retraite. La mise en place d'un droit à l'outplacement pour les 45 ans et plus en Belgique illustre l'accent mis sur l'emploi des travailleurs âgés. Le chapitre III explique ainsi comment l'outplacement est progressivement devenu, avec sa généralisation aux 45 ans et plus, une « technique » au service d'une politique publique belge centrée sur la remontée du taux d'emploi des 55-64 ans.

La seconde partie, qui représente les deux tiers de l'ouvrage, développe une analyse sociologique de l'outplacement. Le chapitre IV synthétise le positionnement théorique qui sert de cadre à T. Moulaert : la notion de «gouvernementalité » de Michel Foucault permet d'envisager l'outplacement à la fois comme un instrument de « conduite des conduites » (au sens où la politique publique de vieillissement actif cherche à orienter les comportements des individus) et comme une « conduite de soi » (au sens où il implique un travail sur soi). L'approche pragmatique retenue vise à comprendre les interactions entre les personnes en s'appuyant sur deux outils conceptuels : les régimes d'action de Laurent Thévenot ${ }^{4}$ et les formes de responsabilité de Jean-Louis Genard et Fabrizio Cantelli ${ }^{5}$. Le chapitre V analyse en détail les matériaux recueillis par l'auteur : vingt et une séances collectives d'outplacement et quatorze entretiens de suivi individuel ont été observés dans trois villes aux caractéristiques économiques et sociales contrastées (Charleroi, Liège et Wavre) ; des entretiens ont été réalisés avec vingt des « candidats » en recherche d'emploi observés et vingt-huit « consultants » de cabinets en outplacement. Cette analyse entend répondre à une question centrale : en quoi consiste et que produit l'outplacement pour des personnes de 45 ans et plus incitées à rechercher un emploi par elles-mêmes ? Il en ressort que les consultants ont pour mission principale de faire en sorte que les candidats opèrent un « travail sur soi [eux-mêmes] ». Il s'agit pour les candidats de se motiver, de se projeter, de se créer un réseau. Les consultants organisent pour cela des situations fictives de recherche d'emploi et font utiliser aux candidats divers outils (tests, curriculum vitae [CV], lettres de motivation, tableaux à remplir, recherche d'offres d'emploi, etc.). Ils ont également pour mission de permettre aux candidats d'acquérir une vision du marché du travail qui les fasse basculer de la posture de « demandeur » à celle d' « offreur ». Atteindre l'objectif de l'outplacement (rendre le candidat capable d'agir par lui-même et d'être autonome dans sa recherche d'emploi) conduit dans certains cas les consultants à jouer sur le « registre du proche », pour reprendre le cadre théorique de L. Thévenot, afin de modérer la pression du travail sur soi. Il arrive aussi que certains candidats n'adoptent pas le comportement attendu, « ne jouent pas le jeu ». En analysant ces contre-conduites difficiles à gérer - à partir des modalités inversées de la responsabilité (ne pas devoir, ne

4. Trois régimes sont distingués : le « régime de familiarité » ou « du proche », dans lequel l'action relève de l'intime et vise à s' accommoder de l'environnement dans lequel on se trouve ; le « régime d'action normale » ou « convenable », dans lequel l'action se matérialise par la conception et la réalisation de plans, de listes, afin d'atteindre des objectifs précis ; enfin, le « régime de justification », dans lequel l'action consiste à critiquer ou à se justifier en mobilisant des argumentations générales.

5. Ces formes de responsabilité se déclinent en « devoir », « vouloir », « savoir » et « pouvoir ». 
pas savoir, etc.) -, T. Moulaert permet de saisir la diversité des situations individuelles et les difficultés pour ces personnes à s'engager dans l'action attendue. Le chapitre VI met en discussion les politiques en faveur du vieillissement actif et les transformations de l'État. Il souligne ainsi les ambiguïtés de l'outplacement belge : redonner au sujet les capacités d'agir (empowerment) passe par sa responsabilisation accrue (activation) sans que soient modifiées les caractéristiques de son environnement. D'un point de vue théorique, la conception du sujet et la place donnée aux outils (CV, fiches, etc.) ouvrent deux zones de dialogue entre approche critique et approche pragmatique de l'action publique. La conclusion générale prolonge cette discussion et dresse trois perspectives de réflexion sur les politiques de vieillissement actif : élargir le champ du vieillissement actif (aux pratiques préventives, à d'autres domaines que l'emploi), analyser l'outplacement en tant que « fabrique » de la responsabilité et replacer le sujet au cœur de l'action publique.

En mobilisant de façon centrale la notion de gouvernementalité, T. Moulaert conduit une réflexion stimulante sur l'évolution de l'action publique en faveur du vieillissement actif, qu'il permet d'analyser à différents niveaux et sous différents angles. Le concept de « gouvernement à distance » (ici de « conduite des conduites ») permet à la fois de rendre compte de la délégation par l'État à des intermédiaires/ professionnels du secteur privé de missions jusqu'alors accomplies par les services publics, et d'illustrer la distance que peuvent prendre les professionnels de l'outplacement à l'égard des injonctions de l'État et des comportements des personnes suivies. L'utilisation du concept de « conduite de soi » permet quant à lui de prendre en compte la personne en tant que sujet, d'envisager les implications et limites de la responsabilité individuelle et d'une mobilisation accrue du travail sur soi. L'étude des interactions entre les personnes et les professionnels dans cette « conduite de soi » enrichit indéniablement la compréhension de la mise en œuvre locale de l'action publique. Les matériaux recueillis illustrent notamment les ambiguïtés de l'outplacement étendu aux travailleurs plus âgés. Si le dispositif est a priori cadré, les conduites des candidats et des conseillers montrent la place (nécessaire) laissée à des ajustements négociés entre candidat et consultant, aux réappropriations par les candidats de ce qui leur est demandé et de ce qu'ils peuvent faire. Il révèle le décalage entre, d'une part, les prescrits et attentes normatives des politiques publiques et, d'autre part, ce qui est fait en pratique.

Le grand sérieux des analyses menées par l'auteur fait regretter que l'ouvrage ne soit pas plus facile d'accès et plus pédagogique pour des lecteurs non familiers du cadre d'analyse retenu. On aurait par ailleurs pu souhaiter une contextualisation plus fine de la genèse de l'outplacement en Belgique. Il est par exemple difficile de saisir précisément les modalités de construction et le statut juridique de la convention collective de travail examinée (qui s' apparente à un accord national interprofessionnel en France). Enfin, il est dommage qu'un certain nombre d'éléments sur la démographie et sur l'évolution de l'outplacement en Belgique n'aient pas fait l'objet d'une mise à jour 
au moment de la publication de l'ouvrage, alors que l'auteur a publié plusieurs articles depuis sa thèse qui auraient pu utilement nourrir certains passages de l'ouvrage ${ }^{6}$.

Cela étant, l'analyse que livre T. Moulaert permet de se confronter à une combinaison de références théoriques pas forcément familières. Et surtout elle contribue à montrer les limites de l'outplacement en tant qu'instrument d'une politique de vieillissement actif : présupposer que l'acteur est capable d'agir ne signifie pas qu'il dispose des ressources nécessaires pour le faire. L'ouvrage invite ainsi à réfléchir à la prise en compte de la diversité des populations par les politiques publiques de vieillissement actif.

6. Par exemple Moulaert T., Léonard D. (2011), « Le vieillissement actif sur la scène européenne », Courrier hebdomadaire du Crisp, n ${ }^{\circ} 2105$, pp. 5-33. 